\title{
On the use of a modified square-well model for prediction and correlation of thermal diffusion factors in binary liquid mixtures
}

Richard L. Rowley

rowley@byu.edu

S. C. Yi

Follow this and additional works at: https://scholarsarchive.byu.edu/facpub

Part of the Chemical Engineering Commons

\section{Original Publication Citation}

Yi, S. C. and R. L. Rowley. "On the use of a modified square-well model for prediction and correlation of thermal diffusion factors in binary liquid mixtures." The Journal of Chemical Physics 87 (1987): 7214-722

\section{BYU ScholarsArchive Citation}

Rowley, Richard L. and Yi, S. C., "On the use of a modified square-well model for prediction and correlation of thermal diffusion factors in binary liquid mixtures" (1987). Faculty Publications. 741.

https://scholarsarchive.byu.edu/facpub/741 


\title{
On the use of a modified square-well model for prediction and correlation of thermal diffusion factors in binary liquid mixtures
}

\author{
S. C. Yi and R. L. Rowley \\ Department of Chemical Engineering, Brigham Young University, Provo, Utah 84602
}

(Received 13 July 1987; accepted 26 August 1987)

\begin{abstract}
Recently reported thermal diffusion factors obtained from heats of transport have been used to test the ability of existing theories to predict thermal diffusivities using model parameters regressed from pure component transport data. None of the theories tested were able to provide even qualitative agreement with experiment. However, predictions using a square-well Enskog theory, modified here for consistency with normal experimental definitions, were found to be approximately correct using pure-component square-well parameters regressed from viscosity and thermal conductivity and an assumed mixing rule for cross terms. Predicted values are very sensitive to the energy mixing rule, and correlation of experimental data is possible for some systems using this theory with one adjustable parameter in the mixing rule.
\end{abstract}

\section{INTRODUCTION}

In a companion paper to this one, ${ }^{1}$ we reported measurement of heats of transport in binary mixtures containing carbon tetrachloride and benzene, toluene, 2-propanone, $n$-hexane, and $n$-octane at 298.15 and $308.15 \mathrm{~K}$ at ambient pressure. A previous study ${ }^{2}$ reported heats of transport for mixtures of carbon tetrachloride with certain straight- and branched-chain $\mathrm{C} 6$ through $\mathrm{C} 8$ paraffins. Heats of transport represent the magnitude of the heat flux produced by molecular diffusion of the constituents in response to a chemical potential gradient. Using thermodynamic information concerning the composition dependence of the activity coefficient in these mixtures and Onsager reciprocity, we calculated thermal diffusion factors from the measured heats of transport. As cross-transport coefficients, thermal diffusion factors (or equivalently, heats of transport) have been considered by some $\mathrm{e}^{3,4}$ to be extremely sensitive to the molecular interactions in solution. Thus, simulation of thermal-diffusion or heat-of-transport data may lead to refined models for intermolecular potential wells or better values and mixing rules for their parameters. The previous two studies provide sufficient data to provide preliminary tests of existing models.

\section{THEORIES FOR THE THERMAL DIFFUSION FACTOR}

Several theoretical approaches have been advanced for the heat of transport and/or thermal diffusion factor. Based on Kirkwood's Brownian motion method, Bearman et al. ${ }^{5}$ developed an expression for heats of transport for systems in which (1) particles react with central forces only, (2) intermolecular potentials can be written as sums of pair potentials, and (3) both components possess only translational energy. The heat of transport for such a system in the absence of external fields is

$$
Q_{1}^{*}=Q_{11}^{*}+Q_{12}^{*} \text {, }
$$

where $Q_{1}^{*}$ is the heat of transport, $Q_{11}^{*}$ is a term involving averages over equilibrium ensembles, and $Q_{12}^{*}$ involves perturbations of the equilibrium distribution due to the flow of heat and matter. The expressions for $Q_{11}^{*}$ and $Q_{12}^{*}$ are complex, but Bearman et al. were able to simplify them for the particular case of regular solutions. Under this simplification the expressions are

$$
\begin{aligned}
Q_{11}^{*}= & \frac{1}{2}\left(\frac{m_{1} x_{1}}{m_{2}}+x_{2}\right)\left(\frac{\bar{v}_{1} \bar{v}_{2}}{\bar{v}}\right)\left(\frac{\bar{L}_{2}}{\bar{v}_{2}}-\frac{\bar{L}_{1}}{\bar{v}_{1}}\right), \\
Q_{12}^{*}= & \frac{1}{2 v}\left(\frac{D_{2}-D_{1}}{D_{2}+D_{1}}\right)\left(\frac{m_{1} x_{1}}{m_{2}}+x_{2}\right)\left\{\bar{v}_{1} \bar{v}_{2}\left(\frac{\bar{L}_{2}}{\bar{v}_{2}}+\frac{\bar{L}_{1}}{\bar{v}_{1}}\right)\right. \\
& +2 x_{1}\left(\bar{L}_{1} x_{1}-\bar{L}_{2} x_{2}\right)+2 x_{2}\left(\bar{L}_{2} \bar{v}_{2}-L_{2} v_{2}\right) \\
& \left.-\frac{2}{3} \int r\left(\frac{d \phi_{12}}{d r}-1\right) V_{21}^{\prime} g_{21}^{\prime} d^{3} r\right\},
\end{aligned}
$$

where $\bar{v}$ is the mean molecular volume, $\bar{v}$ is the partial molecular volume of component $i, v_{i}$ is the molecular volume of pure component $i, \bar{L}_{i}$ is the partial enthalpy departure function of component $i$ relative to the ideal gas state, $L_{i}$ is the enthalpy departure function of pure component $i$ relative to the ideal gas state, $m_{i}$ is the molecular mass of component $i$, $D_{i}$ is the self-diffusion coefficient of component $i$ in the mixture, and $x_{i}$ is mole fraction of component $i$. The integral in Eq. (3) has never been evaluated for real systems because it contains the radial distribution function $g_{12}^{\prime}$, the derivative of intermolecular potential with respect to position $V_{21}^{\prime}$, and the nonequilibrium radial distribution function $\phi_{12}$.

An elementary transition state theory (ETST) has also been developed by Mortimer and Eyring ${ }^{6}$ for calculation of thermal diffusion factors $\alpha$. In terms of the standard state molar enthalpy $\Delta H_{i}^{\ddagger}$ and entropy $\Delta S_{i}^{\ddagger}$ changes for component $i$ in the activation process as molecule 1 moves from the initial uniform state to the high-potential-energy transition state, Mortimer and Eyring write

$\alpha^{(\mathrm{ETST})}=\frac{\Delta H_{1}^{\ddagger} e^{-\Delta H_{\dagger}^{\ddagger}}-\Delta H_{2}^{\ddagger} e^{\left(\Delta S_{2}^{\ddagger}-\Delta S_{1}^{\ddagger}\right) / R} e^{-\Delta H_{2}^{\ddagger} / R T}}{R T\left[x_{2} e^{-\Delta H_{\dagger}^{\ddagger}}+x_{1} e^{\left(\Delta S_{2}^{\ddagger}-\Delta S_{\dagger}^{\ddagger}\right) / R} e^{-\Delta H_{2}^{\ddagger} / R T}\right]}$.

In this method, the thermal diffusion factor not only depends on temperature and concentration, but also on the activation parameters. Theoretically, the latter three quanti- 
ties can be fitted by regression of three or more values of $\alpha$ as a function of composition and temperature. Unfortunately, the sum of the squares of the residuals appears to be a complicated function of the parameters, containing various saddle points and several relative extrema, and confidence in their physical meaning wanes when they are obtained in this manner.

Guy $^{7}$ proposed the following expression for the thermal diffusion factor

$$
\alpha^{(\text {Guy })}=\frac{M_{2}\left(h_{2}^{E}-h_{1}^{E}\right)}{R T\left(1+\Gamma_{22}\right)},
$$

where $M_{2}$ is the molecular weight of the less dense of the two components, $h_{i}^{E}$ is the partial excess enthalpy of component $i$, and $\Gamma_{i i}$ is the so-called thermodynamic factor which is related to the activity coefficient of component $i$ (relative to pure-component standard state) $\gamma_{i}$ by

$$
\Gamma_{i i}=\left(\frac{\partial \ln \gamma_{i}}{\partial \ln x_{i}}\right)_{T, P}
$$

Guy reported reasonable results using Eq. (6) on a single binary system.

The first kinetic theory for a dense hard-sphere fluid was due to Enskog ${ }^{8}$ who generalized the Boltzmann equation to describe hard-sphere dynamics in the dense regime. This theory, commonly called the standard Enskog theory (or SET), was later extended to binary ${ }^{9}$ and multicomponent ${ }^{10}$ dense hard-sphere fluid mixtures. For a binary,

$$
\begin{aligned}
\alpha^{(\mathrm{SET})}= & \frac{5}{2 g_{12}}\left[\left(n / n_{2}\right) m_{1}^{-1 / 2} K_{1}\left(a_{01} a_{-1-1}-a_{0-1} a_{1-1}\right)\right. \\
& \left.+\left(n / n_{1}\right) m_{2}^{-1 / 2} K_{2}\left(a_{0-1} a_{11}-a_{01} a_{1-1}\right)\right] / \\
& \left(a_{11} a_{-1-1}-a_{1-1}^{2}\right),
\end{aligned}
$$

where the function $K_{i}$ is defined by

$$
K_{i}=1+\left(\frac{12}{5}\right) \sum_{j=1}^{2} b_{i j} g_{i j} M_{i j} M_{j i}
$$

Here,

$$
\begin{aligned}
& b_{i j}=2 \pi n_{j} \sigma_{i j}^{3} / 3, \\
& M_{i j}=\frac{m_{i}}{\left(m_{i}+m_{j}\right)}, \\
& \sigma_{i j}=\frac{1}{2}\left(\sigma_{i}+\sigma_{j}\right), \\
& \rho=m_{1} n_{1}+m_{2} n_{2},
\end{aligned}
$$

$m_{i}$ is molecular mass, $n_{i}$ is number density, $\sigma_{i}$ is the hardsphere diameter of component $i$, and $a_{r s}$ are Sonine polynomial coefficients calculatable from the equations given by Chapman and Cowling. ${ }^{11}$

Unfortunately, SET does not agree with Onsager reciprocity. ${ }^{12}$ Additionally, Kincaid et al. ${ }^{13}$ found that SET led to nonphysical values for the mutual diffusion coefficient. Several authors have resolved this inconsistency by redefining the diffusional driving forces to agree with those commonly used in irreversible thermodynamics definitions. Barajas et al. ${ }^{12}$ obtained a revised Enskog theory (or RET1) for the thermal diffusion factor,

$$
\begin{aligned}
\alpha^{(\mathrm{RET} 1)}= & \left(\frac{M_{1} M_{2}}{M^{2}\left(1+\Gamma_{11}\right)}\right)\left[\alpha^{(\operatorname{SET})}\right. \\
& \left.+\frac{2 \pi}{3}\left(n_{1} \sigma_{1}^{3}-n_{2} \sigma_{2}^{3}+2 \frac{m_{2} n_{2}-m_{1} n_{1}}{m_{1}+m_{2}} \sigma_{12}^{3}\right)\right] .
\end{aligned}
$$

Similarly, Lopez de Haro et al. ${ }^{14}$ obtained a revised Enskog theory (or RET2) for the thermal diffusion factor in terms of the derivative of the chemical potential with respect to composition. In this theory, $\alpha$ is given by

$\alpha^{(\mathrm{RET} 2)}=\frac{m_{1} n^{3}\left\{\Sigma_{k=1}^{2} d_{1,0}^{(k)} / n \Sigma_{j=1}^{2} n_{k}\left(\delta_{k j}+2 \rho b_{k j} M_{k j} g_{k j}\right)-E_{k 2} / P_{2} \Sigma_{p=1}^{2} n_{p}\left(\delta_{p j}+2 \rho b_{p j} M_{p j} g_{p j}-a_{0}^{(1)}\right)\right\}}{\rho n_{1} n_{2} \Sigma_{k=1}^{2} d_{1,0}^{(k)}\left(E_{k 1}-P_{1} / P_{2} E_{k 2}\right)}$,

where

$$
\begin{aligned}
& E_{i j}=\frac{n_{i}}{k T}\left(\frac{\partial \mu_{i}}{\partial n_{j}}\right)_{T, n_{k \neq j}}, \\
& P_{i}=\sum_{j=1}^{2} E_{j i},
\end{aligned}
$$

and $d_{1,0}^{(k)}$ and $a_{0}^{(1)}$ are Sonine coefficients. In this theory, the Sonine coefficients are calculated to third order from the equations given by Lopez de Haro et al. ${ }^{14}$

As mentioned, the thermal diffusion factor is expected to be very sensitive to the form of the intermolecular potential and the values of the parameters therein. The hard-sphere theory represents the simplest evaluation of the Enskog theory, but solutions based on more realistic potentials are desirable. Davis et al. ${ }^{15}$ developed a transport equation for the singlet distribution function which is essentially a modified form of the equation derived by Enskog for dense hard spheres. McLaughlin and Davis $^{16}$ extended the square-well theory to binary mixtures. Following the Chapman-Enskog procedure, McLaughlin and Davis derived for the square-well model (SWM)

$$
\begin{aligned}
\alpha^{(\mathrm{SwM})}= & \left(\frac{5 n}{2}\right)\left[\frac{F_{1}\left(a_{01} a_{-1-1}-a_{0-1} a_{1-1}\right)}{n_{2} m_{2}^{1 / 2}}+\frac{F_{2}\left(a_{0-1} a_{11}-a_{01} a_{1-1}\right)}{n_{1} m_{2}^{1 / 2}}\right] /\left(a_{11} a_{-1-1}-a_{1-1}^{2}\right) \\
& +\frac{n b_{12} \rho \zeta_{12}\left(M_{1}-M_{2}\right)}{n_{2}}
\end{aligned}
$$


with the following definitions:

$$
\begin{aligned}
F_{i}= & 1+\frac{3}{5} b_{i i} \rho \xi_{i i}+\frac{12}{5} M_{1} M_{2} b_{i j} \rho \\
& \times\left[\zeta_{12}+M_{j}\left(M_{j}-M_{i}\right) \chi_{12} / \psi_{12}\right], \\
\psi_{i j}= & 1-\exp \left(\epsilon_{i j} / k T\right)+\left(\epsilon_{i j} / 2 k T\right) \zeta_{i j}, \\
\zeta_{i j}= & 1+\frac{4 e^{\left(\epsilon_{i j} / k T\right)}}{\pi^{1 / 2}} \int_{\epsilon_{i j} / k T}^{\infty} e^{-x^{2}} d x, \\
\chi_{i j}= & \frac{\epsilon_{i j}}{\pi^{1 / 2} k T}\left[\frac{J_{1}}{2}-\frac{J_{3}}{3}-\frac{J_{1} \epsilon_{i j}}{3 k T}\right], \\
J_{n}= & \int_{0}^{\infty} x^{n} \sqrt{x^{2}+\epsilon_{i j} / k T} e^{-x^{2}} d x, \\
\zeta_{i j}= & g_{i j}\left(\sigma_{i j}^{(1)}\right)+\left(\frac{\sigma_{i j}^{(2)}}{\sigma_{i j}^{(1)}}\right)^{3} g_{i j}\left(\sigma_{i j}^{(2)}\right) \psi_{i j},
\end{aligned}
$$

where $g_{i j}\left(\sigma_{i j}^{(1)}\right)$ is the value of the radial distribution function evaluated just inside the potential well, $g_{i j}\left(\sigma_{i j}^{(2)}\right)$ is the value just outside the well, and $\epsilon_{12}$ is the well depth between unlike species. In Eq. (17), the $a_{r s}$ are Sonine polynomial coefficients for the square-well model which are calculatable to first order from the equations given by McLaughlin and Davis. $^{16}$

\section{RESULTS USING EXISTING MODELS}

The efficacy of the various models discussed above was tested by comparison of calculated values to those obtained from the experimental work previously reported. ${ }^{1,2}$ In all but Guy's model, potential parameters are required for an assumed intermolecular potential model. This presents two problems: (1) comparison to experiment inseparably tests both the theory and the intermolecular potential model, and (2) intermolecular potential parameters must be available. The first situation means that disagreement with experimental data does not distinguish between failure of the theory and inappropriate choice of the pair potential model. The second situation means that the theory can be used to either correlate data (by regressing potential parameters from the experimental data) or to predict properties (by regressing potential parameters from some other property and using those values in prediction of the desired property). We test the equations in a predictive capacity; parameters in the models are evaluated from other properties.

Bearman's method cannot be evaluated in its entirety because of the nonequilibrium radial distribution function appearing in Eq. (3). While a complete test of this method cannot be made, the equilibrium contribution may yield at least qualitatively correct predictions. Thus calculations were made for $Q_{1}^{*}$ from Eqs. (1) and (2) where the partial molal quantities were evaluated from compiled data. ${ }^{17}$ Table I shows the results obtained from this procedure in comparison to experimental values. ${ }^{1}$ Bearman and Horne ${ }^{18}$ also assumed that $Q_{12}^{*}$ is negligible in their calculations and found that calculated values were too low by $50 \%$ or more. Our results for the systems shown in Table I are in agreement with the findings of Bearman and Horne that the equilibrium portion is generally only a fraction of the experimental value, and it may even have the wrong sign.

The absolute reaction rate theory calculations require activation parameter data as shown in Eq. (4). To retain this as a predictive method, $\Delta H_{1}^{\ddagger}, \Delta H_{2}^{\ddagger}$, and $\left(\Delta S_{2}^{\ddagger}-\Delta S_{1}^{\ddagger}\right)$ were regressed from the model's viscosity equation. ${ }^{19}$ This assumes that the activation parameters are the same for viscous flow and diffusional processes. The results of the calculations are shown in Table I. In this case, the magnitudes are approximately right, but the signs are opposite those of the experimental data. It should be emphasized that disagreement of theory and experiment in this case only implies that predictions of $\alpha$ cannot be made from viscosity data. We do not imply that the model is wrong, only that the activation parameters cannot be obtained from other data and used to predict heats of transport.

The model proposed by Guy is of interest because of its simplicity. However, unlike all of the other models, it contains no nonequilibrium information. We believe that the heat of transport is physically inseparable from the diffui-

\begin{tabular}{|c|c|c|c|c|c|c|c|}
\hline$w_{1}$ & $\alpha^{(\exp )}$ & $\alpha^{(\text {Bearman })}$ & $\alpha^{(\mathrm{ETST})}$ & $\alpha^{\text {(Guy) }}$ & $\alpha^{(\mathrm{SET})}$ & $\alpha^{(\text {RET1 } 1)}$ & $\alpha^{(\text {SWM })}$ \\
\hline \multicolumn{8}{|c|}{ Carbon tetrachloride (1) + benzene $(2)$} \\
\hline 0.1 & 2.41 & -0.028 & -1.98 & 0.100 & 0.44 & -0.28 & 1.06 \\
\hline 0.3 & 2.25 & -0.028 & -1.86 & 0.088 & 0.42 & -0.26 & 1.14 \\
\hline 0.5 & 1.70 & -0.027 & -1.72 & 0.062 & 0.39 & -0.24 & 1.22 \\
\hline 0.7 & 1.16 & -0.027 & -1.58 & 0.010 & 0.36 & -0.20 & 1.30 \\
\hline 0.9 & 0.74 & -0.026 & -1.41 & -0.097 & 0.33 & -0.15 & 1.38 \\
\hline \multicolumn{8}{|c|}{ Carbon tetrachloride $(1)+n$-hexane (2) } \\
\hline 0.1 & .2 .55 & 0.054 & $\cdots$ & 0.261 & 0.35 & -0.79 & 4.71 \\
\hline 0.3 & 2.52 & 0.056 & $\cdots$ & 0.228 & 0.34 & -0.71 & 5.04 \\
\hline 0.5 & 2.15 & 0.059 & $\cdots$ & 0.193 & 0.34 & -0.65 & 5.46 \\
\hline 0.7 & 1.93 & 0.062 & $\cdots$ & 0.051 & 0.34 & -0.56 & 5.96 \\
\hline 0.9 & 1.59 & 0.068 & $\cdots$ & -0.367 & 0.33 & -0.53 & 6.25 \\
\hline
\end{tabular}
sonal mechanism in terms of the energy distributions of the components ${ }^{20}$; a view which is inconsistent with this model. Calculations of $\alpha$ using Eq. (5) were made using $h^{E}$ data obtained from Christensen et al. ${ }^{21}$ Activity coefficient data

TABLE I. Predictions of liquid thermal diffusion factors at $298.15 \mathrm{~K}$ and ambient pressure by various theories in comparison to experimental values 
for Eq. (6) were obtained from the Wilson equation with parameters obtained from vapor liquid equilibrium data. ${ }^{22}$ The results are again shown in Table I. Values obtained from this model are inconsistent with experimental data. Not only is the magnitude of the predictions in error, but the sign is also sometimes wrong.

Application of the various Enskog or kinetic models in a predictive mode requires that intermolecular pair-potential parameters be regressed from other data. For hard-sphere calculations, we regress the pure-component hard-sphere diameters from pure-component thermal conductivity data in accordance with the Enskog model for thermal conductivity. ${ }^{23}$ Values obtained were $5.43,5.27$, and $6.06 \AA$ for carbon tetrachloride, benzene, and $n$-hexane, respectively. The mixing rule for hard spheres, Eq. (11), is used for $\sigma_{i j}$. Using Eq. (7) for SET and Eq. (13) for RET1, we again compare calculated and experimental values in Table I. All $\alpha$ values were about an order of magnitude too small for both models, while RET 1 values were also of the wrong sign. The low SET results are consistent with the low SET predictions found by Palyvos et $a l .^{24}$ for the argon + krypton system. Values for RET2 are not shown in Table I due to the difficulties in accurately evaluating the $E_{i j}$ and $P_{i}$ defined in Eqs. (15) and (16). Calculations were made using experimental data and the Wilson equation to obtain the chemical potentials, but the results were very poor.

From Table I, it would seem that SWM [Eqs. (17)(23) ] is more promising than the hard-sphere models due to its more realistic potential. However, SWM was arrived at in the usual SET manner. Thus, it employs diffusional driving forces which are inconsistent with those used in the irreversible thermodynamics definitions of transport coefficients. Values calculated from these equations would not, therefore, be consistent with experimental values. To make predictions using the square-well model, we first fitted pure component $\epsilon$ and $\sigma$ values by simultaneous regression of pure-component thermal conductivity and viscosity data using the square-well model for those properties given by Reed and Gubbins. ${ }^{25}$ Cross-interaction terms were calculated using an average mixing rule for $\sigma$ and a geometric mixing rule for $\epsilon$ with the ratio of the well distances fixed at 1.8 as shown below:

$$
\begin{aligned}
& \sigma_{i j}^{(k)}=\left(\sigma_{i i}^{(k)}+\sigma_{j j}^{(k)}\right) / 2, \\
& \epsilon_{i j}=k\left(\epsilon_{i j} \epsilon_{j j}\right)^{1 / 2}, \\
& \sigma_{i j}^{(2)} / \sigma_{i j}^{(1)}=1.8 .
\end{aligned}
$$

Values of pure-component square-well potential parameters obtained in this study are shown in Table II.

It is evident from Table I that with the possible exception of SWM no existing model can be used satisfactorily for prediction of $\alpha$.

\section{MODIFICATION OF THE SQUARE-WELL MODEL}

We have modified the square-well model in the same manner that Barajas et al. ${ }^{12}$ modified SET for hard spheres. Using their definition of the diffusional driving force we obtain
TABLE II. Square-well potential parameters regressed from pure component thermal conductivity and viscosity data at $298.15 \mathrm{~K}$ and ambient pressure.

\begin{tabular}{lcc}
\hline \multicolumn{1}{c}{ Component } & $\sigma^{(1)}(\AA)^{\mathrm{a}}$ & $\epsilon / k(\mathbf{K})$ \\
\hline Carbon tetrachloride & 2.21 & 122.7 \\
Chloroform & 4.62 & 307.5 \\
Acetone & 2.03 & 127.5 \\
Benzene & 2.14 & 123.7 \\
Toluene & 2.28 & 121.0 \\
$n$-hexane & 5.85 & 228.1 \\
$n$-heptane & 6.15 & 252.7 \\
$n$-octane & 6.20 & 304.7 \\
3-methylpentane & 5.81 & 226.5 \\
2,3-dimethylpentane & 6.02 & 250.1 \\
2,2,4-trimethylpentane & 6.16 & 288.6 \\
\hline \hline
\end{tabular}

${ }^{\mathrm{a}} \sigma^{(2)}=1.8 \sigma^{(1)}$.

$$
\begin{aligned}
& \alpha^{(\text {MSWM })} \\
& =\left(\frac{M_{1} M_{2}}{M^{2}\left(1+\Gamma_{11}\right)}\right)\left[\alpha^{(\text {SWM })}+\frac{2 \pi}{3}\left(n_{1} \sigma_{11}^{(1)^{3}}-n_{2} \sigma_{22}^{(1)^{3}}\right.\right. \\
& \left.\left.\quad+2 \frac{m_{2} n_{2}-m_{1} n_{1}}{m_{1}+m_{2}} \sigma_{12}^{(1)^{3}}\right)\right]
\end{aligned}
$$

for the modified-square-well model (MSWM), where $\alpha^{\text {(SWM) }}$ represents Eqs. (17)-(23). This expression is exactly analogous to Eq. (13).

While Lebowitz ${ }^{26}$ has solved the Percus-Yevick equation for a mixture of hard spheres, there is no exact expression for the radial distribution function of square-well fluids. For contact encounters in a hard-sphere binary mixture, the radial distribution functions are

$g_{i i}^{\mathrm{hs}}\left(\sigma_{i i}^{(1)}\right)=\frac{\left[1+0.5 \xi+1.5 \eta_{j} \sigma_{i j}^{(1)^{2}}\left(\sigma_{i i}^{(1)}-\sigma_{i j}^{(1)}\right)\right]}{(1-\xi)^{2}}$,

$g_{i j}^{\text {hs }}\left(\sigma_{i j}^{(1)}\right)=\left[\sigma_{i i}^{(1)} g_{i i}\left(\sigma_{i i}^{(1)}\right)+\sigma_{j j}^{(1)} g_{j j}\left(\sigma_{i j}^{(1)}\right)\right] / 2 \sigma_{i j}^{(1)}$,

in which

$$
\xi=\sum_{i=1}^{2} \eta_{i} \sigma_{i i}^{(1)^{3}}
$$

and

$$
\eta_{i}=\pi n_{i} / 6
$$

In the square-well theory, ${ }^{27}$ these hard-sphere functions are augmented by an exponential factor

$$
g_{i j}^{\mathrm{sw}}\left(\sigma_{i j}^{(1)}\right)=g_{i j}^{\mathrm{hs}}\left(\sigma_{i j}^{(1)}\right) \exp \left(\epsilon_{i j} / k T\right) .
$$

The functions $g_{i j}\left(\sigma_{i j}^{(2)}\right)$ are complicated to compute. Throop and Bearman ${ }^{28}$ have performed numerical calculations for these functions and found that for liquid densities

$$
0.8<g_{i j}^{\mathrm{hs}}\left(\sigma_{i j}^{(2)}\right)<1.1 \text {. }
$$

As a result of this apparently limited range over which $g_{i j}^{\text {hs }}\left(\sigma_{i j}^{(2)}\right)$ may vary, the approximation

$$
g_{i j}^{\mathrm{hs}}\left(\sigma_{i j}^{(2)}\right)=1.0
$$

should introduce only small errors into the computed transport coefficients. Sample calculations indicate that this error does not exceed about $3 \%$. Consequently, the approximation represented by Eq. (34) is used in all of our square-well computations. 


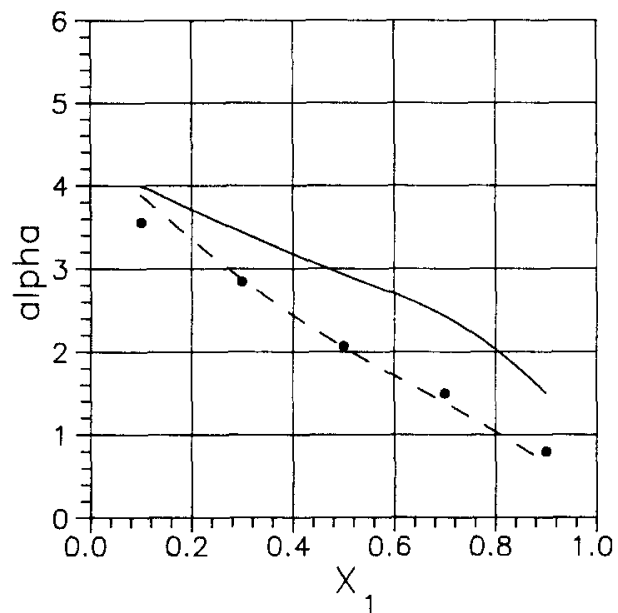

FIG. 1. Experimental $(\bullet)$ and predicted $(-, k=1 ;-, k=1.68)$ values of the thermal diffusion factor for the carbon tetrachloride (1) + acetone (2) system at $298.15 \mathrm{~K}$ and ambient pressure.

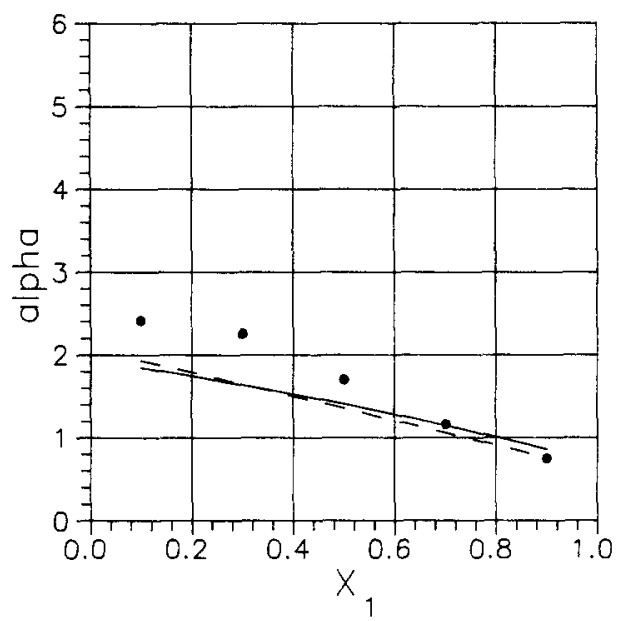

FIG. 2. Experimental $(\bullet)$ and predicted $(-, k=1 ;--, k=1.15)$ values of the thermal diffusion factor for the carbon tetrachloride $(1)+$ benzene (2) system at $298.15 \mathrm{~K}$ and ambient pressure.

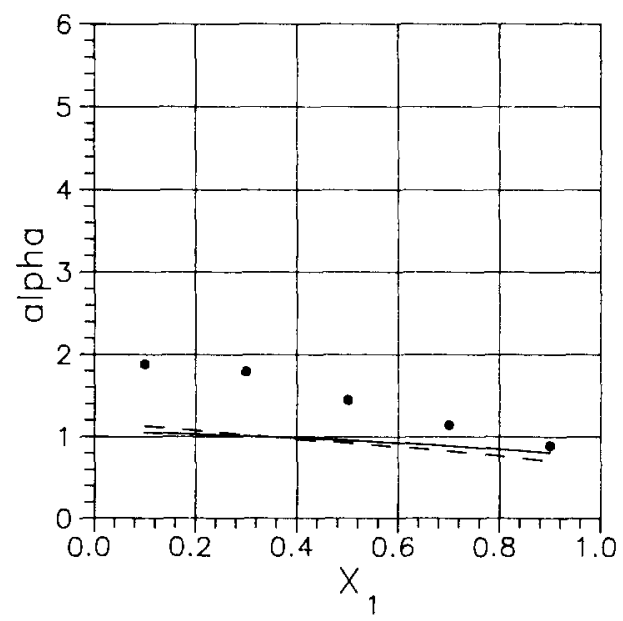

FIG. 3. Experimental $(\bullet)$ and predicted $(-, k=1 ;---, k=1.14)$ values of the thermal diffusion factor for the carbon tetrachloride (1) + toluene (2) sytem at $298.15 \mathrm{~K}$ and ambient pressure.

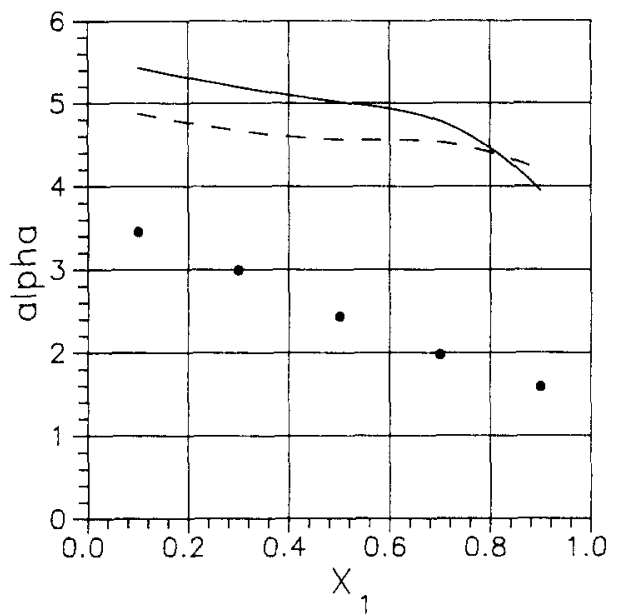

FIG. 4. Experimental (๑) and predicted $(-, k=1 ;--, k=0.92)$ values of the thermal diffusion factor for the carbon tetrachloride (1) $+n$-hexane (2) system at $298.15 \mathrm{~K}$ and ambient pressure.

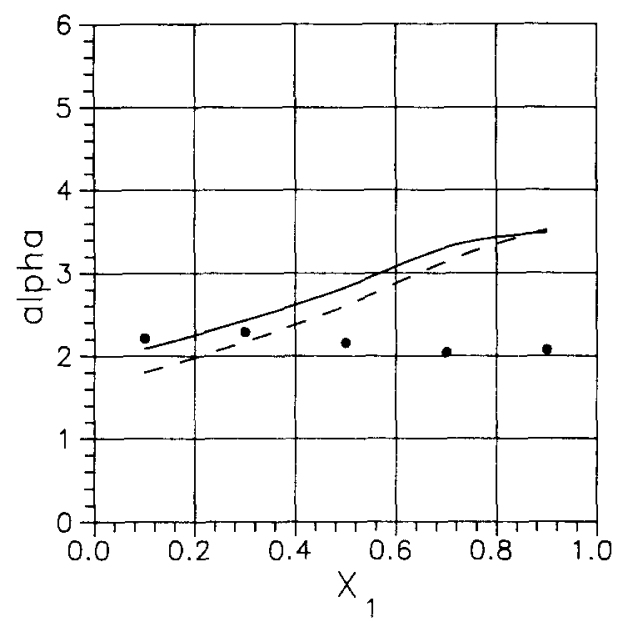

FIG. 5. Experimental $(\bullet)$ and predicted $(-, k=1 ;-\cdots, k=0.95)$ values of the thermal diffusion factor for the carbon tetrachloride (1) $+n$-heptane (2) system at $298.15 \mathrm{~K}$ and ambient pressure.

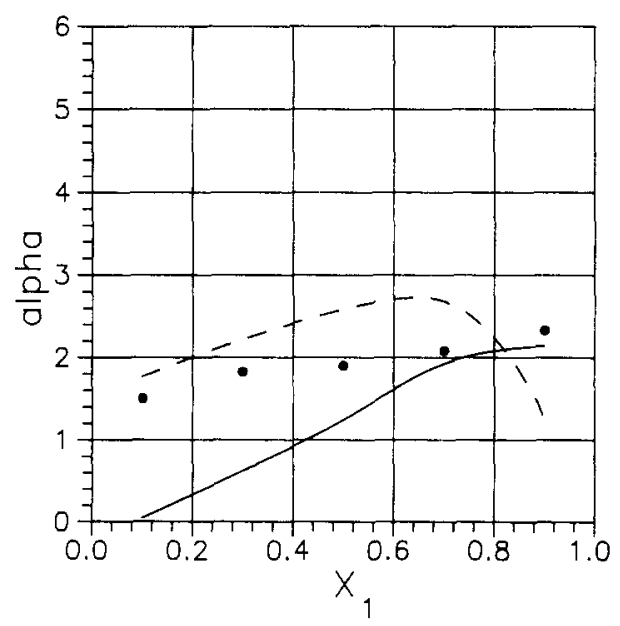

FIG. 6. Experimental ( $)$ and predicted $(-, k=1 ;--, k=1.30)$ values of the thermal diffusion factor for the carbon tetrachloride (1) $+n$-octane (2) system at $298.15 \mathrm{~K}$ and ambient pressure. 


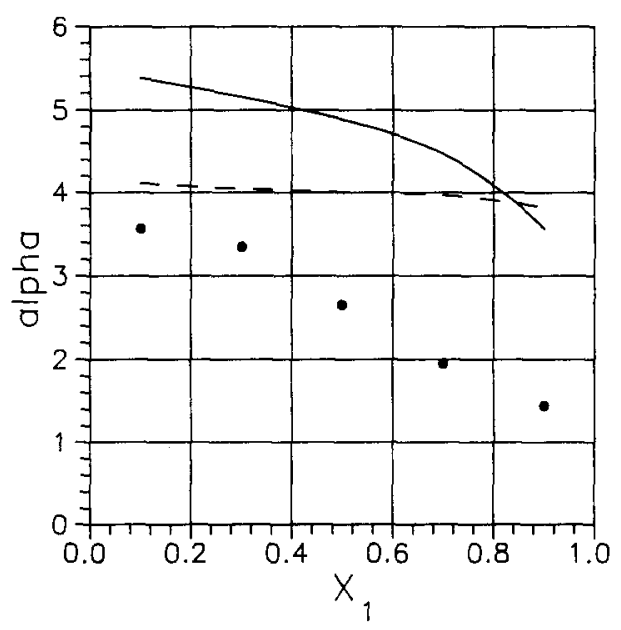

FIG. 7. Experimental ( $)$ and predicted $(-, k=1 ;---, k=0.80)$ values of the thermal diffusion factor for the carbon tetrachloride $(1)+3$-methylpentane (2) system at $298.15 \mathrm{~K}$ and ambient pressure.

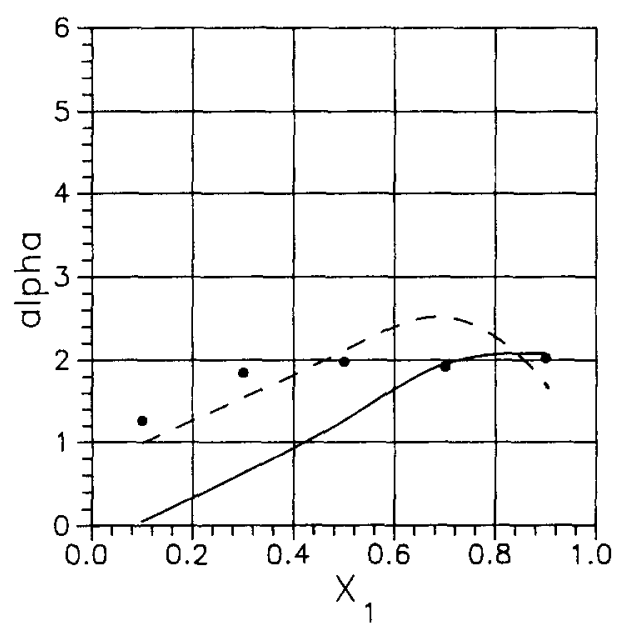

FIG. 8. Experimental $(\bullet)$ and predicted $(-, k=1 ;--, k=1.18)$ values of the thermal diffusion factor for the carbon tetrachloride $(1)+2,2,4$-trimethylpentane (2) system at $298.15 \mathrm{~K}$ and ambient pressure.

\section{DISCUSSION OF RESULTS}

In all of the calculations for this MSWM we used the ad hoc mixing rules of Eqs. (24)-(26). With $k=1$ the method is predictive since all of the constants are obtained from pure component thermal conductivity and viscosity data. The method can also be used in a correlative mode by regressing a value for $k$ in the mixing rule from experimental $\alpha$ values. We have performed both types of calculations and the results are shown in Figs. 1-8.

As a predictive model, MSWM may still be in error by a factor of 2 or more, but it is considerably better than any of the other methods available. It predicts the gross composition dependence of $\alpha$ quite well, and can be used to obtain approximate values. The finer detail of the composition dependence of this model is inherent in the ad hoc mixing rule for $\epsilon$; it is perhaps not surprising that better accuracy is not obtained. As shown in Figs. 1, 2, 6, and 8, considerable flexibility is provided by adjusting the mixing rule for $\epsilon$, and reasonable agreement can be obtained in some cases when $k$ is regressed from the experimental data. The values of $\alpha$ are indeed quite sensitive to the cross term of the energy parameter, and measurement of thermal diffusion factors or heats of transport can provide useful information in refinement of a potential model. The ability of this model to correlate and predict $\alpha$ using parameters obtained from pure transport properties suggests the importance of more extensive studies on other systems in an attempt to develop a general mixing rule.

\section{CONCLUSIONS}

We have used recently measured heat-of-transport data in the form of thermal diffusion factors to test existing models and theories. None of the existing methods were capable of even qualitatively predicting thermal diffusion factors using parameters regressed from pure component transport properties. This is not necessarily an indictment of those theories since there is no way to distinguish between failure of the model and of the assumed potential. However, we have modified the square-well model of Davis in accordance with the technique used by Barajas so that $\alpha$ agrees with the usual experimental definition. Using this method and square-well potential parameters regressed from pure component transport properties, we were able to obtain approximate agreement with experiment. It was found that calculated $\alpha$ values are very sensitive to the mixing rule used for $\epsilon$. This implies that heat-of-transport or thermal-diffusion experiments can be used as a sensitive probe of intermolecular potentials. In particular, it was found that slight adjustment of a constant in the mixing rule for $\epsilon$ enabled improved correlation of measured $\alpha$ values for about half the systems studied.

\section{ACKNOWLEDGMENT}

Support of this project under National Science Foundation Grant No. CBT-85-1424 is gratefully acknowledged.

'S. C. Yi and R. L. Rowley, J. Chem. Phys. 87, 7208 (1987).

${ }^{2}$ R. L. Rowley, S. C. Yi, V. Gubler, and J. M. Stoker, Fluid Phase Equilib. (in press).

${ }^{3}$ J. O. Hirschfelder, C. F. Curtiss, and R. B. Bird, Molecular Theory of Gases and Liquids (Wiley, New York, 1954), p. 582.

${ }^{4}$ E. A. Mason, R. J. Munn, and F. J. Smith, Discuss. Faraday Soc. 40, 27 (1965).

${ }^{5}$ R. J. Bearman, J. G. Kirkwood, and M. Fixman, Advances in Chemical Physics (Interscience, New York, 1958), Vol. 1, pp. 1-13.

${ }^{6}$ R. G. Mortimer and H. Eyring, Proc. Natl. Acad. Sci. U.S.A. 77, 4 (1980).

${ }^{7}$ A. G. Guy, Int. J. Thermophys. 7, 563 (1986).

${ }^{8}$ D. Enskog, K. Sven. Vetenskapsaked. Handl. 63, 4 (1922).

${ }^{9} \mathrm{H}$. H. Thorne, University of Sydney, Australia, as reported in Ref. 11.

${ }^{10}$ M. K. Tham and K. E. Gubbins, J. Chem. Phys. 55, 1 (1971).

${ }^{11} \mathrm{~S}$. Chapman and T. G. Cowling, The Mathematical Theory of Non-Uniform Gases (Cambridge University, New York, 1953).

${ }^{12}$ L. Barajas, L. S. Garcia-Colin, and E. Pina, J. Stat. Phys. 7, 161 (1973).

${ }^{13}$ J. M. Kincaid, E. G. D. Cohen, and M. Lopez de Haro, J. Chem. Phys. 86, $963(1987)$.

${ }^{14}$ M. Lopez de Haro, G. D. Cohen, and J. M. Kincaid, J. Chem. Phys. 78, 5 (1983).

${ }^{15}$ H. T. Davis, S. A. Rice, and J. V. Sengers, J. Chem. Phys. 35, 2210 (1961).

${ }^{16}$ I. L. McLaughlin and H. T. Davis, J. Chem. Phys. 45, 6 (1966).

${ }^{17}$ J. A. Riddick and W. B. Bunger, Techniques of Chemistry, Vol.II. Organic Solvents (Wiley-Interscience, New York, 1970).

${ }^{18}$ R. J. Bearman and F. H. Horne, J. Chem. Phys. 42, 2015 (1965). 
${ }^{19}$ S. Whitaker and R. L. Pigford, Ind. Eng. Chem. 7, 1026 (1958).

${ }^{20}$ R. L. Rowley and F. H. Horne, J. Chem. Phys. 71, 3841 (1979).

${ }^{21} \mathrm{C}$. Christensen, J. Gmehling, P. Rasmussen, and U. Weidlich, Heats of Mixing Data Collection (Dechema, Frankfurt, 1984)

${ }^{22}$ J. Gmehling and U. Onken, Vapor-Liquid-Equilibrium Data Collection; Dechema Chem. Data Series, Vol. 1 (Verlag and Druckerel Friedrich Bischoff, Frankfurt, 1977).

${ }^{23}$ J. H. Ferziger and G. H. Kaper, Mathematical Theory of Transport Pro- cesses in Gases (North-Holland, Amsterdam, 1972).

${ }^{24}$ J. Palyvos, K. D. Luks, I. L. MeLaughlin, and H. T. Davis, J. Chem. Phys. 47, 2082 (1967).

${ }^{25}$ T. M. Reed and K. E. Gubbins, Applied Statistical Mechanics (McGrawHill, New York, 1973).

${ }^{26}$ J. L. Lebowitz, Phys. Rev. A 895, 133 (1964).

${ }^{27}$ H. T. Davis and K. D. Luks, J. Phys. Chem. 69, 869 (1965).

${ }^{28} \mathrm{G}$. J. Throop and R. J. Bearman, J. Chem. Phys. 42, 7 (1965). 